\title{
Hemodinâmica da curva de Langfitt
}

\author{
Marcos Augusto Stávale Joaquim¹, Gustavo Cartaxo Patriota ${ }^{1}$, André de Macedo Bianco² \\ Instituto de Neurociências, São Paulo, SP. \\ Serviço de Neurocirurgia do Hospital Nove de Julho, São Paulo, SP.
}

\section{RESUMO}

Os autores discutem as modificações da hemodinâmica encefálica que conferem à curva de Langfitt o aspecto exponencial que se relaciona com a rápida piora da hipertensão intracraniana. Concluíram ser interessante considerar que a cascata vasodilatadora pode ser importante no desenvolvimento desse fenômeno.

\section{PALAVRAS-CHAVE}

Circulação cerebral. Curva de Langfitt. Hipertensão intracraniana.

\section{ABSTRACT}

The Langfitt curve

The authors discuss the modification in the intracranial hemodynamic that causes the exponential aspect of the ascending part of the Langfitt curve. The hypothesis concerning the importance of the vasodilatation's cascade in the genesis of the phenomenon is very interesting.

\section{KEY WORDS}

Brain circulation. Langfitt curve. Intracranial hypertension.

\section{Introdução}

À medida que uma massa cresce dentro do espaço craniano, uma proporcional quantidade de líquido cefalorraquidiano é retirada dessa caixa. Nesse período, a pressão intracraniana (PIC) não se eleva. Deveríamos esperar que, quando todo compartimento liquórico fosse extraído de dentro do crânio, a curva que determina a relação entre a PIC e o volume acrescido ao espaço - curva pressão/volume - assumisse um caráter retilíneo, e a PIC fosse aumentando linearmente à medida que a massa continuasse crescendo. Entretanto, tal curva assume um traçado gráfico exponencial denotando uma rápida ascensão da PIC após a fase em que todo o liquor foi expulso do compartimento em questão. Nessa fase de ascensão rápida, pequenos acréscimos de volume causam variações positivas da PIC cada vez maiores para um mesmo volume acrescentado. No caso dos inchaços, reperfusionais ou não, esses acréscimos de volume são volêmicos sanguíneos, ou seja, intravasculares. ${ }^{1,17,19,20,24,25,27,38}$
Muito provavelmente, como veremos à frente, tal ascensão exponencial relaciona-se a um acúmulo progressivo de volume sanguíneo intracraniano associado à vasodilatação isquêmica desproporcionalmente grande em relação ao aumento da massa que vem crescendo. Provavelmente, esse aumento de volume sanguíneo é que proporciona o aparecimento da fase exponencial ascendente da curva de Langfitt. Esse acúmulo progressivo de volume sanguíneo encefálico estaria certamente relacionado ao desencadeamento e à persistência da cascata vasodilatadora, já citada em publicações prévias. ${ }^{1,24,25,27}$

\section{Curva de Langfitt}

A curva de Langfitt compreende o traçado que relaciona o eixo da PIC, na vertical, e o eixo do volume que é acrescido à caixa craniana, na horizontal (Figura 1). Nota-se que, no início, o aumento da massa não implica

1. Instituto de Neurociências, São Paulo, SP.

2. Serviço de Neurocirurgia do Hospital Nove de Julho, São Paulo, SP. 
aumento da PIC, mas a partir de uma fase intermediária, chamada de fase de descompensação, a curva assume um caráter exponencial rapidamente ascendente, e a pequena variação de volume acrescida à caixa craniana implica grande aumento de sua pressão interna. Essa curva tem a relação clínica com o fato de que doentes que vêm piorando gradativamente apresentam repentinamente acentuada piora do quadro clínico em razão do rápido aumento da PIC. Na fase inicial da curva ascendente, assim chamada de fase de descompensação, aparecem, no traçado de monitorização da PIC, as ondas patológicas do tipo A, ou seja, aumentos espontâneos e graves da PIC que atingem uma plataforma e, também espontaneamente, retornam à linha de base. Com a evolução do processo, essas ondas tornam-se mais duradouras, mais amplas e mais frequentes, até que praticamente se unem desencadeando a fase ascendente exponencial da curva. .,6,10,12,24,25,27 $^{27}$

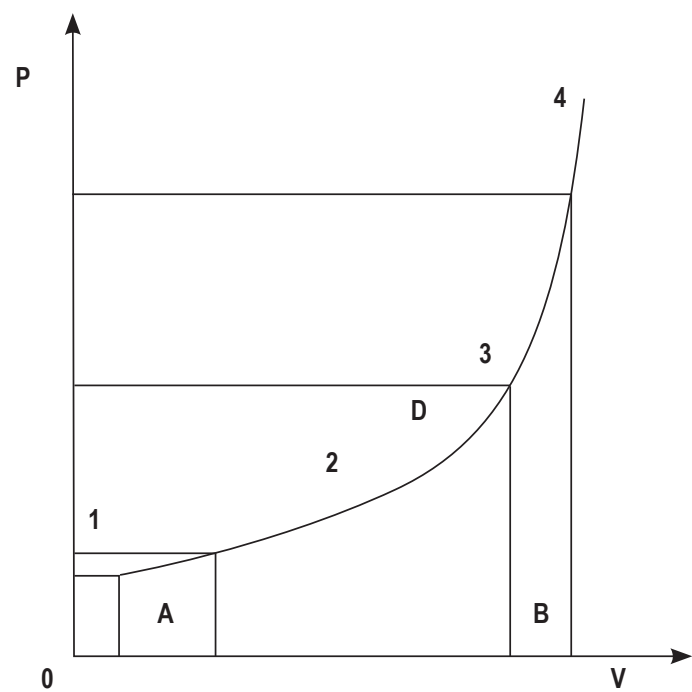

Figura 1 - Curva de Langfitt: No início (A) variações de volume (V) não elevam a PIC (P). Depois, pequenas variações de $V$ elevam bastante a P. Notam-se as fases 1, 2, 3 e 4 .

Nessa fase de descompensação inicial, o liquor já foi extruído da caixa craniana, e variações de volume sanguíneo encefálico são responsáveis pelo aparecimento das ondas patológicas, que se devem a surtos de vasodilatação isquêmica e são revertidas por aumentos reflexos e consecutivos da pressão arterial sistêmica que aumentam a pressão de perfusão encefálica (reflexos de Cushing) e que reperfundem o cérebro isquêmico numa fase em que o mecanismo de autorregulação ainda está preservado, ou seja, ainda existe capacidade contrátil da arteríola pré-capilar, pois a acidose tecidual não é tão intensa. Revertendo-se tal vasodilatação isquêmica e diminuindo- -se o volume sanguíneo encefálico, diminui-se a PIC. $\mathrm{O}$ aparecimento dessas ondas mostra que a complacência intracraniana já tende a zero (Figura 2).,29,13,23-28,30,31,38
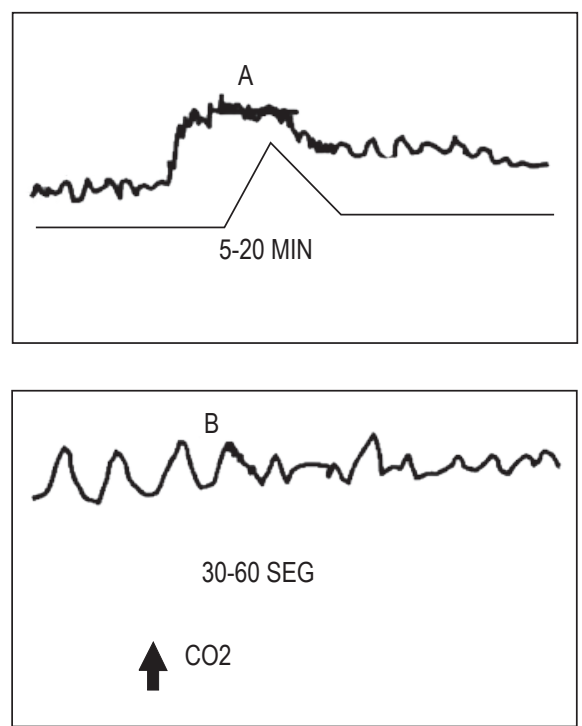

Figura 2-Ondas patológicas: ondas A em plataforma geram aumentos da pressão arterial (linhas retas) e se desfazem; ondas $B$ acompanham os ciclos respiratórios apnêusticos, como o ritmo de Cheyne-Stokes, em suas fases alternadas de hiper e hipoventilação.

\section{Fases modificadas}

$\mathrm{Na}$ curva de Langfitt, a primeira fase pode ser interpretada de maneira pouco distinta da descrita tradicionalmente na literatura. Tal fase diz respeito ao fato de o aumento da massa ser acompanhado de extrusão proporcional de volume liquórico intracraniano. Nessa fase, a PIC não aumenta e também não aumenta o volume sanguíneo cerebral, a não ser que a lesão desencadeante provoque por si um aumento inicial da volemia encefálica, que, mesmo assim, ainda funcionará como um processo expansivo com o crescimento compensado pela extrusão liquórica, sob o ponto de vista pressórico.

Na segunda fase, a fase de descompensação, todo o liquor intracraniano já foi extruído e o aumento de volume da massa começa a elevar a PIC. No momento em que essa elevação da PIC implicar queda mais significativa da pressão de perfusão encefálica, ocorre isquemia, e sua consequência natural é a vasodilatação encefálica, que tem o intuito de manter o fluxo sanguíneo cerebral à custa de uma diminuição da resistência vascular. Nessa fase, o volume sanguíneo intracraniano (volume sanguíneo encefálico) começa a se elevar; também praticamente não há mais liquor dentro do crânio, e então as variações da PIC serão associadas às variações do volume sanguíneo encefálico. Variações positivas do 
volume sanguíneo devem-se à vasodilatação isquêmica, e variações negativas, à reversão da vasodilatação isquêmica, quando ocorre a reperfusão em tempo adequado. Se pequenas variações de volume sanguíneo encefálico, desencadeadas pela vasodilatação isquêmica e pela vasoconstrição reperfusional, já causam grandes aumentos da PIC, nota-se que a complacência tende a zero e o doente está entrando na fase ascendente da curva de Langfitt, ou seja, a um pequeno acréscimo de volume intravascular associa-se uma importante elevação da PIC. Esse fato é a princípio mostrado na fase de ascensão da PIC nas ondas patológicas e, mais tarde, mostrado pela ascensão definitiva da PIC na fase exponencial ascendente da curva de Langfitt. 24,25,27,31-34

A terceira fase é notada como a fase exponencial ascendente da curva anteriormente descrita. $\mathrm{O}$ volume sanguíneo encefálico passa a aumentar de maneira exponencial ou logarítmica certamente em virtude do desencadeamento da cascata vasodilatadora. A isquemia inicial gerou uma vasodilatação isquêmica com aumento hipervolêmico da PIC, que gerará maior decréscimo da pressão de perfusão, isquemia adicional, vasodilatação adicional, e assim por diante, ocorrendo um aumento em aceleração do volume sanguíneo intracraniano que eleva a pressão do compartimento até que esta se iguale à pressão arterial média, quando o fluxo sanguíneo encefálico será zero.

Essa fase final seria a quarta fase hemodinâmica da curva citada. $3,4,14,15,17,19-21,27,29$

\section{Discussão}

Nesse entendimento, a curva pode ser interpretada puramente em bases hemodinâmicas. Sob o ponto de vista fisiopatológico, na fase inicial, em que não há elevação da PIC ou queda da pressão da perfusão encefálica, não há isquemia, portanto não há vasodilatação, e a extrusão liquórica compensa o volume adicional intracraniano. Nessa fase não há modificações hemodinâmicas. Na fase subsequente já passa a haver queda da pressão de perfusão encefálica com o acréscimo da massa que vinha crescendo inicialmente. Essa queda da pressão de perfusão encefálica implica aparecimento da dilatação isquêmica da arteríola pré-capilar, ou seja, vasodilatação isquêmica e aumento do volume sanguíneo encefálico. A cada onda patológica que se forma, há um novo surto de isquemia e reperfusão, associado a uma acidose tecidual progressiva, cada vez mais difícil de ser tamponada, o que causará a dilatação progressiva arteriolar pré-capilar (o sistema de resistência é controlado pelo $\mathrm{pH}$ periarteriolar) que inundará o sistema de capacitância constituído por microcirculação, vênulas e veias e que contém $70 \%$ do volume sanguíneo encefálico. ${ }^{4,5,7,8,10,11,16,22,36}$

$\mathrm{Na}$ terceira fase, ascendente exponencial, não há mais surtos de isquemia e reperfusão. Existe apenas uma ascensão progressiva da PIC e uma queda progressiva da pressão de perfusão encefálica associada à isquemia progressiva. A isquemia encefálica progressiva gerará mais acidose tecidual com mais vasodilatação e aumento da volemia intracraniana. Nessa fase retoma-se o conceito de que o cérebro isquêmico é um cérebro que possui baixo fluxo associado a alto volume sanguíneo intrínseco. Na quarta fase a pressão de perfusão intracraniana encefálica torna-se nula, pois a PIC atinge os níveis da pressão arterial média. Essa fase corresponde à morte encefálica. ${ }^{21,22,38}$

Sob o ponto de vista de raciocínio hemodinâmico, torna-se clara a importância de preconizar-se que os doentes tenham a PIC monitorizada para que a ascensão de seus valores possa ser prevista já durante a fase de aparecimento das ondas patológicas ao monitor. Essas ondas mostram surtos de vasodilatação e vasoconstrição alternados e associados à nulidade da complacência intracraniana e exigem que o doente seja agressivamente tratado já a partir dessa fase e não apenas quando ele já tiver atingido a fase exponencial ascendente.

Portanto, nota-se que na primeira fase não existem variações patológicas do calibre vascular e da volemia intracraniana. Na segunda fase existe uma alternância entre vasodilatação isquêmica e vasoconstrição reperfusional associadas ao aparecimento das ondas patológicas. Na terceira fase existe apenas uma dilatação vascular progressiva sem associação com a vasoconstrição reperfusional, exceto se o tratamento surtir efeito. Essa vasodilatação progressiva associa-se ao aumento progressivo do volume sanguíneo encefálico que eleva exponencialmente a PIC à custa do desencadeamento da cascata vasodilatadora. Nessa terceira fase a arteríola pré-capilar encefálica estará progressivamente parética, mas ainda não plégica, ou seja, o mecanismo de autorregulação da circulação cerebral estará funcionando de maneira deficiente em virtude da acidose progressiva, mas ainda há capacidade contrátil da arteríola pré-capilar, que se encontra cada vez mais dilatada, mas ainda funcionante. ${ }^{7,8,38}$

Na quarta fase existe plegia da arteríola pré-capilar e inundação da microcirculação. Não há respostas da musculatura lisa da arteríola pré-capilar às manobras terapêuticas que possam interferir com sua capacidade contrátil. Como o sistema está completamente vasodilatado na quarta fase e a PIC está equivalente à pressão arterial média, a pressão de perfusão tenderá a zero e o fluxo sanguíneo cerebral cessa. É interessante notar que o fluxo sanguíneo cerebral está parado porque a pressão arterial média se transferiu à PIC que é igual 
à pressão venosa encefálica. Assim, a pressão arterial média torna-se igual à pressão venosa encefálica, e o gradiente pressórico no sistema tubular tende a zero. Este é o real motivo da parada da circulação cerebral, e não a teoria antiga de que um aumento da PIC comprimiria primeiramente as veias, de parede mais fina, e apenas depois as artérias, de parede mais espessa e mais forte, o que implicaria aparecimento de um período em que o sangue continuaria entrando no crânio, mas não conseguiria mais sair. Essa foi, durante muito tempo, a explicação dada para a existência da fase ascendente da curva de Langfitt. Tal explicação simplista já não mais procede, pois na fase de vasoplegia completa toda circulação intracraniana está dilatada, sem gradiente pressórico arteriovenoso e sem fluxo. ${ }^{20-22,37}$

\section{Conclusão}

Considerando apenas os aspectos hemodinâmicos relacionados à hipertensão intracraniana, podemos concluir que, em relação às quatro fases evolutivas, ocorrem os fenômenos que serão citados.

Na primeira fase, quando o aumento de um volume intracraniano é compensado pela extrusão liquórica, não há variações hemodinâmicas, exceto se o evento inicial, como uma isquemia aguda, já houver causado alterações hemodinâmicas. Entretanto, aqui se estudam apenas as modificações relacionadas à hipertensão intracraniana e não ao evento causador inicial. Nessa fase o volume sanguíneo encefálico não aumenta.

$\mathrm{Na}$ segunda fase, a do aparecimento das ondas patológicas, o doente já está na fase ascendente da curva, com complacência muito pequena que induz à ocorrência de grandes aumentos da PIC por pequenos aumentos do volume sanguíneo encefálico por vasodilatação isquêmica. O mecanismo de autorregulação da circulação cerebral (capacidade contrátil da arteríola pré-capilar) está preservado, pois o reflexo de Cushing dissolve a onda patológica causando vasoconstrição cerebral pelo restabelecimento da perfusão, o que diminui a volemia cerebral e a PIC. Nessa fase, o volume sanguíneo encefálico aumenta e diminui alternadamente nas ondas patológicas. Ainda não se desencadeou a cascata vasodilatadora (Figura 3).

Na terceira fase, de elevação exponencial pura, o volume sanguíneo encefálico aumenta progressivamente junto à PIC e ao reflexo de Cushing, embora presente, não desfaz esse aumento hipervolêmico encefálico da PIC. O mecanismo de autorregulação da circulação cerebral está progressivamente parético. Nessa fase, a volemia encefálica, se não a tratarmos, apenas aumentará. Está desencadeada a cascata vasodilatadora.

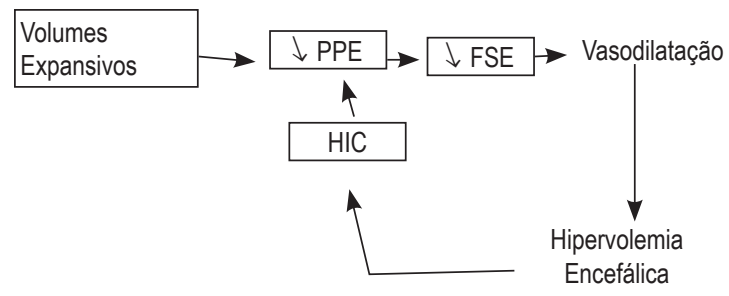

Figura 3 - A cascata vasodilatadora: volumes expansivos diminuem a pressão de perfusão encefálica (PPE) e o fluxo (FSE), gerando vasodilatação, hipervolemia, hipertensão intracraniana (HIC) adicional e mais isquemia por maior decréscimo da PPE. O sistema torna-se um círculo vicioso.

$\mathrm{Na}$ quarta fase, ao final da curva exponencial, o volume sanguíneo encefálico é máximo, a PIC é igual à pressão arterial média, e a pressão de perfusão e o fluxo sanguíneo encefálicos valem zero. É a morte encefálica. No início dessa fase o Doppler transcraniano mostra penetração do sangue no crânio apenas na sístole e depois nem mesmo na sístole, quando a PIC ultrapassa a pressão sistólica.

\section{Referências}

1. Auer LM, Ishiyama N, Pucher R. Cerebrovascular response to intracranial hypertension. Acta Neurochir (Wien). 1987;84:124-8.

2. Auer LM, Sayama I. Intracranial pressure oscillation (B Waves) caused by oscillation in cerebrovascular volume. Acta Neurochir (Wien). 1983;68:93-100.

3. Cruz J, Miner ME, Allen SJ, Alves WM, Gennarelli TA. Continuous monitoring of cerebral oxygenation in acute brain injury: assessment of cerebral hemodynamic reserve. Neurosurgery. 1991;29:743-9.

4. Enevoldsen EM, Cold G, Jensen FT, Malmros R. Dynamic changes in regional $\mathrm{CBF}$, intracranial pressure, CSF $\mathrm{pH}$, and lactate levels during the acute phase of head injury. $J$ Neurosurg. 1976;44:191-214.

5. Fitch W, McDowall EG. Systemic vascular responses to increased intracranial pressure: 1. Effects of progressive intracranial epidural balloon expansion on intracranial pressure and systemic circulation. J Neurol Neurosurg Psychiatry. 1977; 40:833-42.

6. Fitch W, McDowall DG, Keaney MP, Pickerodt VWA. Systemic vascular responses to increased intracranial pressure: The "Cushing" response in the presence of intracranial space-occupying lesions: systemic and cerebral haemodinamic studies in the dog and the baboon. J Neurol Neurosurg Psychiatry. 1977;40:843-52.

7. Gray WJ, Rosner MJ. Pressure-volume index as a function of cerebral perfusion pressure. Parte. I. The effects of cerebral perfusion pressures changes and anesthesia. J Neurosurg. 1987;67:369-76.

8. Gray WJ, Rosner MJ. Pressure-volume index as a function of cerebral perfusion pressure. Part 2. The effects of low cerebral perfusion pressure and autoregulation. J Neurosurg. 1987;67:377-80. 
9. Grubb Jr. RL, Raichle ME, Phelps ME, Patcheson PA. Effects of increased intracranial pressure on cerebral blood volume, blood flow and oxygen utilization in monkeys. J Neurosurg. 1975;43:385-98.

10. Hamer J, Albert F, Hoyer S, Wiedemann K. Influence of systemic and cerebrovascular factors on cerebrospinal fluid pulse waves. J Neurosurg. 1977;46:36-45.

11. Hedges TF, Weinstein JD. Cerebrovascular responses to increased intracranial pressure. J Neurosurg. 1964;21:292-7.

12. Hekmatpanah J. The sequence of alterations in the vital signs during acute experimental increased intracranial pressure. J Neurosurg. 1970;32:16-20.

13. Hekmatpanah J. Cerebral circulation and perfusion in experimental increased intracranial pressure. J Neurosurg. 1970;32:21-9.

14. Johnston IH, Rowan JO. Raised intracranial pressure and cerebral blood flow. 3. Venous outflow tract pressures and vascular resistances in experimental intracranial hypertension. J Neurol Neurosurg Psychiatry. 1974;37:392402.

15. Johnston IH, Rowan JO, Harper AM, Jennet WB. Raised intracranial pressure and cerebral blood flow. 1. Cisterna magna infusion on primates. J Neurol Neurosurg Psychiatry. 1972;35:285-96.

16. Johnston IH, Rowan JO, Park DM, Rennie MJ. Raised intracranial pressure and cerebral blood flow. 5 . Effects of episodic intracranial pressure waves in primates. J Neurol Neurosurg Psychiatry. 1975;38:1076-82.

17. Kato Y, Auer LM. Cerebrovascular response to elevation of ventricular pressure. Acta Neurochir (Wien). 1989;98:184-8.

18. Kjallqvist A, Lundberg N, Ponten U. Respiratory and cardiovascular changes during rapid spontaneous variations of ventricular fluid pressure in patients with intracranial hypertension. Acta Neurol Scand. 1964;40:291-317.

19. Langfitt TW, Kassell NF. Acute brain swelling in neurosurgical patients. J Neurosurg. 1966;24:975-83.

20. Langfitt TW, Kassell NF, Weinstein JD. Cerebral blood flow with intracranial hypertension. Neurology. 1965;15:761-73.

21. Langfitt TW, Weinstein JD, Kasse NF. Cerebral vasomotor paralysis produced by intracranial hypertension. Neurology. 1965;15:622-41.

22. Langfitt TW, Weinstein JD, Kassel NF, Gagliard, LJ, Shapiro HM. Compression of cerebral vessels by intracranial hypertension. I. Dural sinus pressures. Acta Neurochir (Wien). 1966;15:212-22.

23. Lassen NA. The luxury-perfusion syndrome and its possible relation to acute metabolic acidosis localized within the brain. Lancet. 1966;2:1113-5.

24. Leech P, Muller JD. Intracranial volume-pressure relationship during experimental brain compression in primates. 2 . Effect of induced changes in systemic arterial pressure and cerebral blood flow. J Neurol Neurosurg Psychiatry. 1974;37:1099-104.
25. Löfgren J, Von Essen C, Zwetnow NN. The pressure volume curve of the cerebropinal fluid space in dogs. Acta Neurol Scand. 1973;49:557-74.

26. Lorenz R. The Cushing response. In: Beks JWF, Bosch DA, Brock M (Eds.). Intracranial Pressure III. New York: Springer-Verlag, 1976. p.270-8.

27. Lowell HM, Bloor BM. The effect of increased intracranial pressure on cerebrovascular hemodynamics. J Neurosurg. 1971;34:760-9.

28. Marshall LF, Durity F, Lounsbury R, Graham DI, Welsh F, Langfitt TW. Experimental cerebral oligoemia and ischemia produced by intracranial hypertension. Part 1. Pathophysiology, electroencephalography, cerebral blood flow, blood-brain barrier, and neurological function. J Neurosurg. 1975;43:308-16.

29. Miller JD, Stanock A, Langfitt TW. Concepts of cerebral perfusion pressure and vascular compressions during intracranial hypertension. Prog Brain Res. 1971;35:411-32.

30. Miller JD, Stanock A, Langfitt TW. Cerebral blood flow regulations during experimental brain compression. J Neurosurg. 1973;39:186-96.

31. Nemoto EM, Snyder JV, Carroll RG, Morita H. Global ischemia in dogs: cerebrovascular $\mathrm{CO} 2$ reactivity and autoregulation. Stroke. 1075;6:425-31.

32. Risberg J, Lundberg N, Ingvar DM. Regional cerebral blood volume during acute transient rises of the intracranial pressure (plateau waves). J Neurosurg. 1969;31:303-10.

33. Smith DR, Jacobson J, Kobrine AL, Rizzoli HV. Regional cerebral blood flow with intracranial mass lesions. Part I. Local alterations in cerebral blood flow. Surg Neurol. 1977;7:233-7

34. Smith DR, Jacobson J, Kobrine Al, Rizzoli HV. Regional cerebral blood flow with intracranial mass lesions. Part II. Autoregulation in localized mass lesion. Surg Neurol. 1977;7:238-40.

35. Symon L, Branstson NM, Strong AJ. Autoregulation in acute focal ischemia. An experimental study. Stroke. 1981;7:547-54.

36. Symon K, Crockard HA, Juhasz J, Branstorn NM. The effect of intracranial hypertension on cerebrovascular resistance. An experimental study. Acta Neurochir (Wien). 1976;35:121-31.

37. Yada K, Nakagawa Y, Tsuru M. Circulatory disturbance of the venous system during experimental intracranial hypertension. J Neurosurg. 1973;39:723-9.

38. Zwetnow N, Kjällquist A, Siesjo BK. Cerebral blood flow during intracranial hypertension in reation to tissue hipoxia and to acidosis in cerebral extracellular fluids. Prog Brain Res. 1968;30:87-92.

Endereço para correspondência Marcos Augusto Stávale Joaquim Alameda Campinas, $1.360,16^{\circ}$ andar 01404-002 - São Paulo, SP

Email: marcos.stavale@terra.com.br 